\title{
Comparison of Two Methods for The Determination of Potassium in Vitreous Humor
}

\author{
Focardi $\mathbf{M}^{1}$, Lanzilao $\mathrm{L}^{2}$, Bonari $\mathrm{A}^{2}$, Rensi $\mathbf{R}^{1}$, Pinchi $\mathbf{V}^{1}$ and Gualco $\mathrm{B}^{1 *}$ \\ ${ }^{1}$ Department of Health Sciences, University of Florence, Italy
}

${ }^{2}$ Azienda Ospedaliera Universitaria Careggi Florence, Italy

*Corresponding author: Gualco Barbara, Department of Health Sciences, University of Florence, Italy.

To Cite This Article: Gualco Barbara.Comparison of Two Methods for The Determination of Potassium in Vitreous Humor. Am J Biomed Sci \& Res. 2019 - 4(1). AJBSR.MS.ID.000760. DOI: 10.34297/AJBSR.2019.04.000760

Received: July 09, 2019 | Published: July 17, 2019

\begin{abstract}
The estimation of time since death is one of the important issues faced in the daily forensic practice. The well-known and largely adopted methods of investigation in an early phase, however, cannot be applied after the first 24 hours from death. Several body parts and fluids have been analyzed over the years in order to find a correlation between biochemical markers and the postmortem interval. Among those methods, the study of the concentration of potassium in vitreous humor demonstrated a time-related increase. The methods for the determination of potassium concentration published in the literature are of common use in clinical chemistry laboratory, even if we should consider the well-known matrix effect given that such instruments are validated for plasma or urine analysis. The aim of the present research is to compare a gold standard method not affected by this effect with the low cost and easily available indirect potentiometric method provided by an automatized system.
\end{abstract}

Keywords: Potassium; Postmortem interval; Vitreous humor

\section{Introduction}

The estimation of the post-mortem interval (PMI) after the first 24 hours is one of the most studied issues in forensic medicine, as it is crucial for medico-legal investigations. Over the last decades, many efforts were dedicated to the analysis of the biochemical composition of the vitreous humor ( $\mathrm{VH})$, as it is submitted to a slow contamination following autolytic and contamination processes because of its peculiar site-related characteristics [1-3]. In the different researches taken into account from the 1960s [4], Potassium $(\mathrm{K}+)$ concentration largely shows an increase in a timedependent manner. Several authors gave their own equation for the correlation of the PMI to the $\mathrm{K}+$ concentration in $\mathrm{VH}$; moreover it is has been established over the years that the increase may be influenced from several parameters such as temperature, duration of the terminal episode and age of the individual [5-7]. The studies performed on the $\mathrm{VH}$ over the years have already pointed out the importance of the matrix effect, as the analytes, and especially $\mathrm{K}+$, are often determined with instruments validated for serum or urine analysis [8]. To avoid this bias, in our research we wanted to compare the $\mathrm{K}+$ concentration in $\mathrm{VH}$ by means of an automatized instrument adopting a potentiometric low cost and quick method easily available in a clinical chemistry laboratory with one gold standard method not affected from the matrix effect.

\section{Material and Methods}

We selected 120 cadavers with known time of death. Cases in which we found pathological conditions that may alter the concentration of electrolytes in the vitreous humor (ocular trauma, kidney failure, drug overdose, metabolic disorders, hyperhydration or dehydration before death) were excluded, as well as those in which autopsies revealed the presence of chronic diseases. Age and sex were not considered criteria for an exclusion. The vitreous humor samples were obtained 20 to 200 hours after death in the different eyes (right and left) at the same time, with a sterilized 20-gauge hypodermic needle inserted near the center of the eyeball, as reported in literature. Only crystal-clear humor was collected and stored at $-80^{\circ} \mathrm{C}$. Pre-analytical preparation of the samples was made following the requirements of Thierauf et al. [9]. The samples were analyzed in one session adopting protocols on the automated Siemens Dimension Vista analyzer (V-LYTE® Integrated Multisensory K800A), validated for serum, plasma, urine analysis and CSF; the dilution was made in distilled water, in order to fulfill range requirements specified from the manufacturer of the method.

Mass spectrometry (MS) was performed on $1 / 3$ of the samples (randomly chosen) with an iCAPQ inductively coupled plasma 
(ICP)-MS (Thermo Fisher Scientific, Waltham, MA, USA) equipped with a Cetac ASX520 autosampler (Cetac Technologies, Omaha, NE, USA), a PFA cyclonic spray chamber, and a 2.5 - $\mathrm{mm}$ internaldiameter quartz injector. Samples were diluted $1: 5$ in $1 \%$ nitric Acid and $\mathrm{K}+$ levels were processed in collision mode with the following parameters: peristaltic pump speed: $40 \mathrm{rpm}$; nebuliser gas flow rate: $0.91 \mathrm{~L} \mathrm{min-1;} \mathrm{radio} \mathrm{frequency} \mathrm{power:} 1.550 \mathrm{~W}$; cool gas flow: $14 \mathrm{~L} \mathrm{min-1;}$ and auxiliary gas flow: $0.89 \mathrm{~L} \mathrm{min-1}$, collision cell gas (helium) flow - $3.5 \mathrm{ml}$ min-1. Dwell times were $50 \mathrm{~ms}$ for $39 \mathrm{~K}$ and $10 \mathrm{~ms}$ for internal standards(72Ge), with 10 sweeps per replicate and five replicates per sample. Blank wipe samples, spiked wipe samples with only the IS solution, and six calibration standards prepared were adopted to build up a calibration curve at the following concentrations: $2.5,5.0,10.0,20.0,40.0,80.0 \mathrm{mmol} / \mathrm{L}$.

\section{Results}

Among the sample individuals, 108 were male, 12 females; mean age: 56,5 (minimum 20, maximum 90 years); the vitreous humor samples were obtained after a median of 47,5 hours (range from 20 to 200). Right and left VH taken from the same patient did not show any difference; this allowed us to be sure of the good quality of the drawing and of the sample preservation. The comparison between the two analytical methods was performed by linear correlation between $\mathrm{K}+$ concentrations obtained with the potentiometric method and those obtained with the ICP-MS and evaluating the resulting intercept, slope and determination coefficient obtained. The results are shown in Table 1.

\begin{tabular}{|c|c|c|c|c|}
\hline \multicolumn{5}{|c|}{ Table 1: Results of methods used for $\mathrm{K}^{+}$determination. } \\
\hline \multirow{2}{*}{$\mathrm{K}^{+}(\mathrm{mmol} / \mathrm{L})$} & $\mathbf{N}$ & Slope & Intercept & $\mathbf{R 2}$ \\
\cline { 2 - 5 } & 40 & 1,01 & $+0,09$ & 0,92 \\
\hline
\end{tabular}

\section{Discussion}

The estimation of the PMI is crucial for medicolegal investigation, and after the publications of Sturner and Adelson in 1963, over the past fifty years several authors confirmed the PM linear increase of $\mathrm{K}+$ concentration in $\mathrm{VH}$. The most part of the authors did not report any differences of biochemical marker concentration among the two eyeballs in the same patient $[10,11]$, and, apart from the common problem found in the literature due to the difficulty to find information related to pre-existing pathologies of the patient [1213], the finding of a strict relation between analytes concentration of $\mathrm{VH}$ and serum confirms the results of our research and gives us the evidence of the good quality of the withdrawal procedure. Over the years, the importance of pre-analytical treatment was well discussed and several authors analyzed the problem of determining the analytes through automated systems, not validated for such biological fluids [9] and specifically not for the VH, actually affected by different matrix components; among the techniques used for the evaluation of the $\mathrm{K}+$ concentration, capillary electrophoresis was validated by comparing results with flame photometry, even if in most papers the authors adopted automatized instruments validated for other biological fluids without comparing the results with a gold standard method of measurement of the electrolyte concentration [3,8,14-16].
In our work, therefore, after the adoption of all the pre-analytical precautions reported by Thierauf et al. [9], we aimed to compare the results obtained with a simple and low cost automatized instrument for the determination of the $\mathrm{K}+$ concentration, which adopts an indirect potentiometric method and largely available in most analysis laboratories, with the results obtained with ICP-MS which is considered a gold standard. The results were perfectly consistent with a variability comparable with the intra-instrumental one.

\section{Conclusion}

With our results we want to give evidence that a low-cost potentiometric method available in automatized systems used in clinical chemistry laboratories, and validated for other biological fluids, show a variability which is compatible with those obtained with an intra-instrumental method when compared to ICP-MS results.

\section{References}

1. Harper DR (1989) A comparative study of the microbiological contamination of postmortem blood and vitreous humour samples taken for ethanol determination. Forensic Sci Int 43(1): 37-44.

2. Coe JI (1974) Postmortem chemistry: practical considerations and a review of the literature. J Forensic Sci 19(1): 13-32.

3. Tse R (2018) Post mortem vitreous magnesium in adult population. Forensic Sci Int 284: 46-52.

4. Sturner WQ (1963) The vitreous humour: postmortem potassium changes. Lancet 1(7285): 807-808.

5. Komura S, S Oshiro (1977) Potassium levels in the aqueous and vitreous humor after death. Tohoku J Exp Med 122(1): 65-68.

6. Stephens RJ, RG Richards (1987) Vitreous humor chemistry: the use of potassium concentration for the prediction of the postmortem interval. J Forensic Sci 32(2): 503-509.

7. Siddamsetty AK (2014) Estimation of time since death from electrolyte, glucose and calcium analysis of postmortem vitreous humour in semiarid climate. Med Sci Law 54(3): 158-166.

8. Blana SA (2011) Variations in vitreous humor chemical values as a result of pre-analytical treatment. Forensic Sci Int 210(1-3): 263-270.

9. Thierauf A, F Musshoff, B Madea (2009) Post-mortem biochemical investigations of vitreous humor. Forensic Sci Int 192(1-3): 78-82.

10. Mari F, Defraia B, Rensi R (2013) Fatal Poisoning two to co-ingestion of benzodiazepines and alcohol: a forensic approach. Ind J Forensic Med and Tox 1: 45-47.

11. Focardi M, Gualco B, Norelli GA (2008) Accidental Death in Autoerotic Manouvres. Am J Forensic Med and Path. 1: 64-68.

12. Focardi M, Bugelli V, Defraia B (2018) Accidental death in autoerotic maneuvers: case series. Rom J Leg Med 29(1): 258-263.

13. Mari F, Gualco B, Rensi R (2012) Acute Massive Pulmonar Thromboembolism due to acute intoxication by Duloxetine: a case report Card Cardiovasc Toxicol 12(3): 258-262.

14. Madea B, F Musshoff (2007) Postmortem biochemistry. Forensic Sci Int 165(2-3): 165-171.

15. Chandrakanth HV (2013) Postmortem vitreous chemistry--an evaluation of sodium, potassium and chloride levels in estimation of time since death (during the first $36 \mathrm{~h}$ after death). J Forensic Leg Med 20(4): 211216.

16. Mihailovic Z (2012) Estimation of the postmortem interval by analyzing potassium in the vitreous humor: could repetitive sampling enhance accuracy? Am J Forensic Med Pathol 33(4): 400-403. 